\title{
Movement Kinematics and Their Relationship with Performance in Target Acquisition Task Using a Mouse
}

\author{
Yuen-Keen Cheong, ${ }^{1,2}$, Randa L. Shehab ${ }^{1}$, and Chen Ling ${ }^{1}$ \\ ${ }^{1}$ School of Industrial Engineering \\ University of Oklahoma \\ Norman, OK 73019
${ }^{2}$ Consumer Experience Design (CXD)
Motorola Inc.
Libertyville, IL 60048

\begin{abstract}
Movement kinematics has been shown useful for characterizing the process of aiming movement in target acquisition tasks. There are multiple kinematic measures reported in the literature, but their relationship to eventual performance is not well documented. To determine the relationship between various kinematic measures and movement performance, data were collected from participants aged 21 to 90 years with a wide range of psychomotor ability. When computed across age groups, time to peak velocity (TPV), time to peak acceleration (TPA), and time from peak velocity until the end of movement (TPVEND) were found to correlate with movement performance. However, the relationships diminished when the correlations were computed within age groups (except for TPVEND). More interestingly, despite the extensive report, certain kinematic measures such as peak velocity were found to be uncorrelated with performance. Thus, when performance is the focus, improvement should be made to reduce TPV, TPA, and TPVEND.
\end{abstract}

\section{INTRODUCTION}

Endpoint measures such as movement time and target misses are often recorded in a target acquisition task. However, these measures are unable to explain the process of the movement itself. To overcome that limitation, kinematics provide a sound approach for characterizing the process of aiming movement.

Kinematics is a study of motions without considering the forces that may cause the motion. A review of literature showed that analysis of movement kinematics, sometimes also referred to as movement microstructure, is more prevalent in research involving direct aiming movement using a limb (e.g., Carlton, 1994; Chua \& Elliot, 1993; Elliot, Carson, Goodman, \& Chua, 1991; Helsen, Elliot, Starkes, \& Ricker, 1998; and Ricker et al., 1999). However, more recently this approach has been adopted in aiming movement using indirect means such as computer input devices (MacKenzie, Kauppinen, \& Silfverberg, 2001; Mithal \& Douglas, 1996; Slocum, Chaparro, McConnell, \& Bohan, 2005; and Slocum, Thompson, \& Chapparo, 2005).

Most studies of movement kinematics are loosely based on the stochastic optimized submovement (SOS) model (Meyer, Abrams, Kornblum, Wright, \& Smith, 1988; and Meyer, Smith, Kornblum, Abrams, \& Wright, 1990). Present consensus indicates that the SOS model is the most appropriate model for describing the process of aiming movement. According to Elliot, Helsen, \& Chua (2001), the model builds upon previous models; it inherits the feedback-control hypothesis from the deterministic model, as well as the endpoint variability hypothesis of the impulse variability model. Among all, the most recognizable attribute of the SOS model is the notion of neuromotor noise, whereby the model posits that submovement endpoints are normally distributed around the center of the target. Feedback systems (i.e., visual sensory, and kinesthetic sensory to a lesser extent) may detect movement discrepancy (i.e., overshooting or undershooting) and as a result corrective submovements .

While the kinematics of aiming movement can be captured using different kinematic measures, the relationship of these measures to the eventual performance is often not discussed. It is believed that the knowledge of the correlation between certain kinematic characteristics and performance would be valuable for overcoming inefficient mouse use. For instance, an adaptive user interface capable of detecting irregularities in movement kinematics can be used to invoke corrective measures to improve performance of mouse use. Such an idea was elaborated by Hwang, Keates, Langdon, \& Clarkson (2004) in the context of designing mouse input for motor-impaired users, who were found to demonstrate excessive submovements (i.e., pauses) during aiming movement. In this case, a 
novel solution such as cursor stabilization could be activated to assist mouse use.

The objective of this study was to determine the kinematics-performance relationship of aiming movement using a computer mouse. It was hoped that identification of such relationship would enable a deeper understanding of the underlying process of aiming movement, thus pinpointing the specific kinematics for improvement. To increase generalizability, it was determined that data should be collected from subjects across age groups that are representative of a wide range of psychomotor ability.

\section{METHOD}

\section{Participants}

Forty-five healthy participants between 21 and 90 years of age were recruited from a university community, as well as from an independent living center. There were fifteen participants in each age group: younger $(M=25.25$ years old, $S D=2.89)$, middle-aged $(M=50.37$ years old, $S D=5.01)$, and older $(M=80.62$ years old, $S D=6.16)$. Participants were required to have normal or near-normal vision, with correction if necessary. Older participants were screened for dementia using a mini-mental state exam (Folstein, Folstein, \& McHugh, 1975). All were experienced mouse users except for one middle-aged and two older participants. Inexperienced participants were trained to use the mouse.

\section{Procedure}

Participants performed five psychomotor tests and a pointing task. All tasks were performed using the dominant hand. The order of testing was randomized, with a break of about 2 minutes between each task. The psychomotor tests were used to evaluate a number of elemental psychomotor abilities as suggested by Fleishman \& Reiley (1992): precision control (rotary pursuit), arm-hand steadiness (grooved-type steadiness test), manual dexterity (Minnesota manual dexterity test), finger dexterity (Purdue pegboard), and wristfinger speed (tapping board). The pointing task was performed using a mouse under the Fitts' paradigm; IDTest (International Business Machines [IBM], 1999) was used for generating stimuli for the pointing task. The experiment concluded after all tasks were completed.

\section{Experimental Task}

The mouse pointing task was operationalized using a multi-directional reciprocal Fitts' pointing task, similar to that described in Soukoreff \& MacKenzie (2004). The task was performed on a Windows-based laptop computer with 1280-by-800 pixels screen resolution. The pointing device used was a neutral shaped Microsoft Optical Mouse connected to the computer via a universal serial bus (USB) port. The targets were circular in shape with a diameter of 30 pixels and were separated by 50, 100, 200, 400, and 650 pixels. Using Shannon's formulation $\log _{2}((\mathrm{D}+\mathrm{W}) /$ $\mathrm{W})$ ), the combinations produced five conditions with index of difficulty (ID) values ranging from 1.42 to 4.50 bits; the range was approximately half of that (i.e., 2 to 8 bits) recommended by Soukoreff \& MacKenzie (2004). The targets were arranged along various angles (i.e., 0 , 45, 90, and 135 degrees). The task required participants to point the cursor at the target and then acquire it using the left mouse button. The targets became transparent if successfully acquired. However, a beep was audible should the participant miss the intended target. Following a practice trial, each distance condition was tested five times at each angle. Therefore, each participant had 100 trials (i.e., 5 distances $\times 4$ angles $\times 5$ repetitions). The treatments (i.e., distance-angle combinations) were presented in random order for all trials, with a short break between treatments. Movement time (MT) and cursor x-y positions were sampled across time using IDTest. Note the sampling of cursor positions was event-based. Each time a mouse movement was detected, the $x-y$ position would be recorded (B.A. Smith, personal communication, 1 December, 2006).

Cursor displacements were computed from the raw position data using the Pythagorean Theorem. The displacement data were linearly interpolated at $200 \mathrm{~Hz}$, and then smoothed using a fourth order Butterworth lowpass filter with a cut-off frequency of $6 \mathrm{~Hz}$. Data smoothing and the computations of velocities and accelerations were based on the techniques described in Winter (2005). Eight kinematic measures were recorded from the velocity and acceleration profiles: peak velocity (PV), peak acceleration (PA), time to peak velocity (TPV), time to peak acceleration (TPA), proportion of time to peak velocity (PROPTPV), proportion of distance traveled at peak velocity (PROPDPV), proportion of time to peak acceleration (PROPTPA), and time from peak velocity until the end of movement (TPVEND). 


\section{RESULTS AND ANALYSIS}

\section{Participants’ Psychomotor Ability}

A series of one-way ANOVAs was performed to determine age differences in various elemental psychomotor abilities (see Table 1). Age differences were detected in all abilities: precision control $\left(F_{2,42}=\right.$ 8.82, $p=.0006)$, arm-hand steadiness $\left(F_{2,42}=5.90, p=\right.$ $.0055)$, manual dexterity $\left(F_{2,42}=37.26, p=.0001\right)$, finger dexterity $\left(F_{2,42}=27.12, p<.0001\right)$, and wristfinger speed $\left(F_{2,42}=12.24, p<.0001\right)$. Ryan's multiple comparison procedure was performed to determine pairwise differences in age group. The results revealed psychomotor ability in the older age group was significantly lower than the younger and the middleaged groups; there was no significant difference between the younger and the middle-aged.

Table 1. Participants' Elemental Psychomotor Abilities.

\begin{tabular}{|c|c|c|c|}
\hline & \multicolumn{3}{|c|}{ Age Group } \\
\hline & Younger & Middle-Aged & Older \\
\hline \multicolumn{4}{|c|}{ Precision control (time-on-target, in seconds) } \\
\hline$M$ & 11.91 & 11.05 & 6.34 \\
\hline$S D$ & 3.69 & 4.41 & 3.57 \\
\hline \multicolumn{4}{|c|}{ Arm-hand steadiness (error time, in seconds) } \\
\hline$M$ & .31 & .25 & .71 \\
\hline$S D$ & .20 & .19 & .64 \\
\hline \multicolumn{4}{|c|}{ Manual dexterity (completion time, in seconds) } \\
\hline M & 171.16 & 183.86 & 243.17 \\
\hline$S D$ & 13.62 & 24.18 & 31.85 \\
\hline \multicolumn{4}{|c|}{ Finger dexterity (number of pins inserted) } \\
\hline$M$ & 17.07 & 16.20 & 12.03 \\
\hline$S D$ & 1.87 & 1.60 & 2.44 \\
\hline \multicolumn{4}{|c|}{ Wrist-finger speed (number of taps) } \\
\hline$M$ & 62.87 & 58.50 & 45.98 \\
\hline$S D$ & 9.50 & 9.44 & 10.15 \\
\hline
\end{tabular}

\section{Movement Profiles}

A velocity profile was drawn for each Fitts' trial, producing 4500 profiles (i.e., 5 distances $\times 4$ angles $\times 5$ repetitions $\times 45$ participants). Visual examination on selected profiles revealed that the younger group produced a higher PV in a shorter time (i.e., TPV) than the middle-aged and the older groups (see Figure 1). In addition, a longer TPVEND was observed in the older group as opposed to the other two groups (see Figure 1); this was accompanied by the higher number of secondary submovements (i.e., zero crossings) in the older group (see Figure 2).

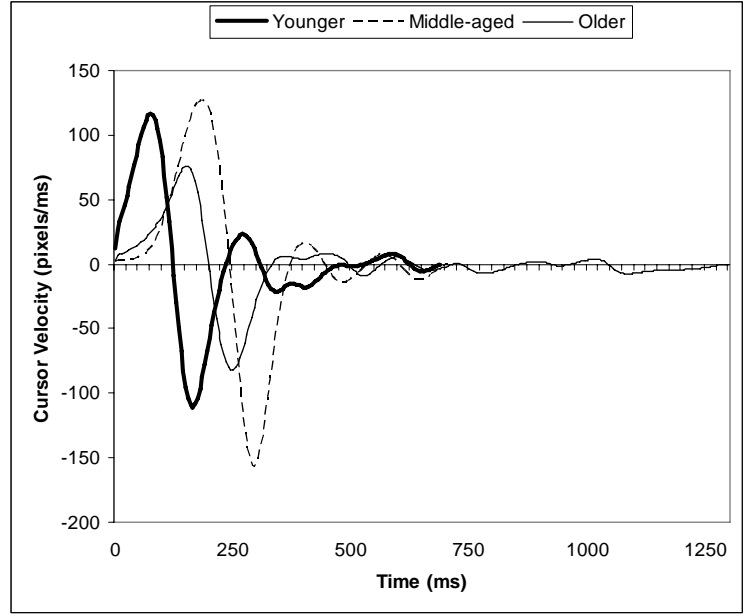

Figure 1. Selected Individual Velocity Profiles.

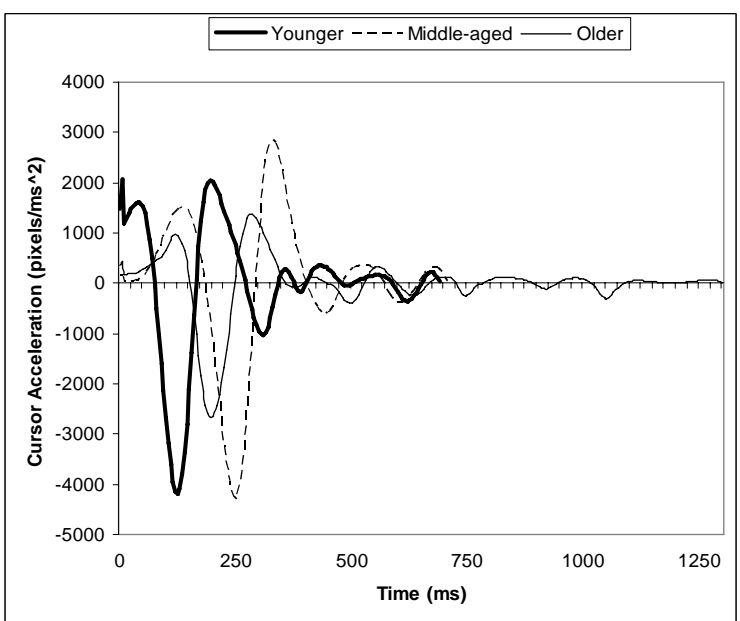

Figure 2. Selected Individual Acceleration Profiles.

\section{Overall Kinematics-Performance Correlations}

Overall bivariate correlations (i.e., across all age groups) were computed for each pair of the kinematic measures and movement time (MT), using sample size $N$ $=4500$ (i.e., 100 trials $\times 45$ participants). The correlations were found as follows: PV-MT $(r=.07)$, PA-MT $(r=.16)$, TPV-MT $(r=.68)$, TPA-MT $(r=.61)$, PROPDPV-MT $(r=.24)$, TPVEND-MT $(r=.96)$, PROPTPV-MT ( $r=-.05)$, PROPTPA-MT $(r=-.18)$. It was determined that only correlations with a magnitude larger than |.35| were of practical value; those smaller than $\mid .35$ | were considered minimally correlated. Based on that criterion, only TPV, TPA, and TPVEND were strongly correlated with MT.

\section{Within-Group Kinematics-Performance Correlations}

It was hoped that further insight could be uncovered by analyzing the kinematics-MT relationship 
within each age group. Specifically, age differences in regard to the correlation magnitudes of TPV-MT, TPAMT, and TPVEND-MT were of interest. Other correlations were excluded because the magnitudes were negligible. For each distance condition (i.e., over all angles and repetitions), kinematics-MT correlations were computed for each participant. Referring to Table 2, the within-age group correlations were generally negligible, except for TPVEND-MT where strong correlations were observed in all age group.

Table 2. Within-Age Group Kinematics-Performance Correlations ( $r$ ).

\begin{tabular}{lccc}
\hline \multirow{2}{*}{ Pair of Variables } & \multicolumn{3}{c}{ Age Group } \\
\cline { 2 - 4 } & Younger & Middle-Aged & Older \\
\hline TPV-MT & .26 & .24 & .31 \\
TPA-MT & .14 & .14 & .29 \\
TPVEND-MT & .94 & .93 & .89 \\
\hline
\end{tabular}

\section{DISCUSSION}

The objective of this study was to investigate the kinematics-performance relationship of pointing movement performed using a computer mouse. Data were collected from participants of three age groups (i.e., younger, middle-aged, and older). The ANOVAs demonstrated that the participants represented a wide range of psychomotor ability with the older group as having significantly poorer psychomotor ability. Based on visual examination of selected movement profiles, age differences were noted in the movement kinematics. Overall analysis revealed only certain kinematic measures (i.e., time to peak velocity (TPV), time to peak acceleration (TPA), and time from peak velocity until the end of movement (TPVEND)) to be correlated with performance. When disaggregated by age, some of these correlations became inconsequential, suggesting that the age effect drove the correlations rather than the inherent relationship between the kinematic and performance measures.

While kinematics-performance relationships are rarely documented in the literature, some authors (e.g., Elliot et al., 1991; and Slocum, Chapparo, et al., 2005) suggested that PV, TPV, and PROPDPV as a predictor for movement efficiency. Except for TPV, results from this study could not establish the relationship of PV and PROPDPV with performance. Furthermore, despite being the most reported kinematic measure in the literature, peak velocity (PV) was found uncorrelated with performance. A similar finding was also reported in Ketcham, Seidler, Van Gemmert, \& Stelmach (2002), whereby PV was discounted as a major contributor to performance. Interestingly, correlation of TPV-MT and
TPA-MT computed within age group were minimal and no strong relationship could be established; the observed overall correlation seemed to be driven by between group differences rather than within group differences. Hence, the finding implies that the relationship between primary submovement and movement time could be mediated by the age effect, and that the causal relationship between primary submovement and performance (i.e., MT) is not as profound as it seems. As for TPVEND-MT, the correlation was consistently strong in either way (i.e., between and within); thus it implies that the homing time (i.e., TPVEND) is more influential to performance.

Results from this study can be explained using the notion of feedback control in the stochastic optimized submovement (SOS) model. According to the model, visual feedback is not as critical during the ballistic phase (i.e., primary submovement). Other functional abilities such as motor control (e.g., ability to exert momentum onto the mouse) and psychomotor ability play a larger role than visual feedback. The similarity of psychomotor ability within age group may explains the minimal TPV-MT and TPA-MT correlations computed within age groups. On the contrary, the effect of varying psychomotor ability between age group is demonstrated by the profound TPV-MT and TPA-MT correlations computed across age groups. It is therefore speculated that the kinematics-performance relationship during the ballistic phase could be mediated by the psychomotor ability. As for the homing phase, Chua \& Elliot (1993) and Elliot et al. (1991) showed this phase is highly influenced by visual feedback. In this study, the vision aspect was a controlled factor because all participants, irrespective of age groups had at least near-normal vision. Thus, TPVEND-MT could be thought as a direct causal relationship because even though vision was essentially controlled, profound correlation was detected.

In the context of designing input devices such as a computer mouse, a common strategy for improving performance is by manipulating the parameters in Fitts' law (i.e., target width and distance). Such manipulation has been limited to novel enhancement of the graphical user interface (see Balakrishnan, 2004). Now that pertinent kinematic measures have been identified, hardware enhancement can be devised to facilitate movement kinematics, and consequently improving mouse use. For instance, a stabilizing mechanism could be used to reduce the fluctuation of cursor position when acquiring a target on the screen (see Hwang et al., 2004).

Perhaps the most interesting finding of this study is that not all kinematic measures are corrrelated with performance, despite being reported extensively in 
the literature. Nevertheless, they can be useful for characterizing the process of pointing movement. A number of other studies have reported kinematic differences along a host of different dimensions, such as age (e.g., Ketcham et al., 2002), index of difficulty (e.g., Ketcham et al., 2002), eye-hand coordination (e.g., Helsen et al., 1998), and type of input devices (e.g., Slocum, Chaparro, et al., 2005). Finally, it is cautioned that this study was conducted using a computer mouse and the kinematics-performance relationship identified here may not be generalizable to a different pointing device.

Finally, two related variables (i.e., device control order and control-display (C/D) gain) were not considered in this study. For most input devices, the gain ratio is not constant and it changes according to the order of control for that device. A rate-control mouse moves the cursor at a greater length if its displacements are sped up. Despite the common belief, empirical studies (i.e., Kantowitz \& Elvers, 1988; and Lin, Radwin, \& Vanderheiden, 1992) have found no evidence of gain setting effect on performance. However, gain setting was reported in Thompson, McConnell, Slocum, \& Bohan (2007) as having an effect on PV and PROPDPV. On the question of whether gain setting affects other kinematic measures reported in this study, it remains to be answered by future studies.

\section{REFERENCES}

Balakrishnan, R. (2004). “Beating” Fitts’ law: virtual enhancements for pointing facilitation. International Journal of HumanComputer Studies, 61(6), 857-874.

Carlton, L.G. (1994). The effects of temporal-precision and timeminimization constraints on the spatial and temporal accuracy of aimed hand movements. Journal of Motor Behavior, 26(1), 43-50.

Chua, R., \& Elliot, D. (1993). Visual regulation of manual aiming. Human Movement Science, 12, 365-401.

Elliot, D., Carson, R.G., Goodman, D., \& Chua, R. (1991). Discrete vs. continuous visual control of manual aiming. Human Movement Science, 10, 393-418.

Elliot, D., Helsen, W.F., \& Chua, R. (2001). A century later: Woodworth's (1899) two-component model fo goal-directed aiming. Psychological Bulletin, 127(3), 342-357.

Fleishman, E.A. \& Reily, M.E. (1992). Handbook of Human Abilities: Definitions, Measurements, and Job Task Requirements. Palo Alto, CA: Consulting Psychologists Press.

Folstein, M.F., Folstein, S.E., \& McHugh, P.R. (1975). Mini-mental state: A practical Method for Grading the Cognitive State of Patients for the Clinician. Journal of Psychiatric Research, 12, 189-198.

Helsen, W.F., Elliot, D., Starkes, J.L., \& Ricker, K.L. (1998). Temporal and spatial coupling of point of gaze and hand movements in aiming. Journal of Motor Behavior, 30(3), 249259.
International Business Machines [IBM]. (1999). IDTest (Version 2.3) [Computer software]. New York: Author.

Kantowitz, B.H., \& Elvers, G.C. (1988). Fitts' law with an isometric controller: Effects of order of control and control display gain. Journal of Motor Behavior, 20(1), 53-66.

Ketcham, C., Seidler, R., Van Gemmert, A., \& Stelmach, G. (2002). Age-related kinematic differences as influenced by task difficulty, target size, and movement amplitude. Journal of Gerontology: Psychological Sciences, 57B(1), 54-64.

Lin, M.L., Radwin, R.G., \& Vanderheiden, G.C. (1992). Gain effects on performance using a head-controlled computer input device. Ergonomics, 35(2), 159-175.

MacKenzie, I.S., Kauppinen, T., \& Silfverberg, M. (2001). Accuracy measures for evaluating computer pointing devices. Proceedings of the SIGCHI Conference on Human Factors in Computing Systems, 9-16.

Meyer, D.E., Abrams, R.A., Kornblum, S., Wright, C.E., \& Smith, J.E.K. (1988). Optimality in human motor performance: Ideal control of rapid aimed movements. Psychological Review, 95(3), 340-370.

Meyer, D.E., Smith, J.E.K., Kornblum, S., Abrams, R.A., \& Wright, C.E. (1990). Speed-accuracy tradeoffs in aimed movements: Toward a theory of rapid voluntary action. In M. Jeannerod (Ed.), Attention and Performance: Vol. XIII (pp. 173-226). Hillsdale, NJ: Lawrence Erlbaum.

Mithal, A.K., \& Douglas, S.A. (1996). Differences in movement microstructure of the mouse and the finger-controlled isometric joystick. Proceedings of the SIGCHI Conference on Human Factors in Computing Systems, 300-307.

Ricker, K.L., Elliot, D., Lyons, J., Gauldie, D., Chua, R., \& Byblow, W. (1999). The utilization of visual information in the control of rapid sequential aiming movements. Acta Psychologica, 103, 103-123.

Slocum, J., Chapparo, A., McConnell, D., \& Bohan, M. (2005). Comparing computer input devices using kinematic variables. Proceedings of the Human Factors and Ergonomics Society 49th Annual Meeting, 711-715.

Slocum, J., Thompson, S., \& Chapparo, B. (2005). Evaluation of mouse pads designed to enhance gaming performance. Proceedings of the Human Factors and Ergonomics Society 49th Annual Meeting, 706-710.

Soukoreff, R.W., \& MacKenzie, I.S. (2004). Towards a standard for pointing device evaluation, perspectives on 27 years of Fitts' law research in HCI. International Journal of HumanComputer Studies, 61, 751-789.

Thompson, S.G., McConnell, D.S., Slocum, J.S., \& Bohan, M. (2007). Kinematic analysis of multiple constraints on a pointing task. Human Movement Science, 26, 11-26.

Winter, D.A. (2005). Biomechanics and Motor Control of Human Movemen (3rd ed.). Hoboken, NJ: John Wiley \& Sons. 